Bioorg Med Chem Lett. 2012 June 15; 22(12): 4018-4022. doi:10.1016/j.bmcl.2012.04.084.

\title{
Design and synthesis of gambogic acid analogs as potent cytotoxic and anti-inflammatory agents
}

\author{
Chiao-Ting Yen ${ }^{a, b}$, Kyoko Nakagawa-Goto ${ }^{a}$, Tsong-Long Hwang ${ }^{c}$, Susan L. Morris-

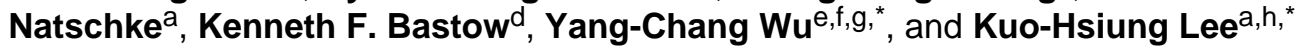 \\ aNatural Products Research Laboratories, UNC Eshelman School of Pharmacy, University of \\ North Carolina at Chapel Hill, NC 27599 7568, USA \\ ${ }^{b}$ Graduate Institute of Natural Products, Kaohsiung Medical University, Kaohsiung 807, Taiwan \\ 'Graduate Institute of Natural Products, Chang Gung University, Taoyuan 333, Taiwan \\ ${ }^{d}$ Division of Chemical Biology and Medicinal Chemistry, UNC Eshelman School of Pharmacy, \\ University of North Carolina, Chapel Hill, NC 27599 7568, USA \\ 'Center for Molecular Medicine, China Medical University Hospital, Taichung 40447, Taiwan \\ ${ }^{f}$ Natural Medicinal Products Research Center, China Medical University Hospital, Taichung \\ 40447, Taiwan \\ 9School of Chinese Medicine, College of Chinese Medicine, China Medical University, Taichung \\ 40402, Taiwan \\ ${ }^{\mathrm{h}}$ Chinese Medicine Research and Development Center, China Medical University and Hospital, \\ Taichung 40447, Taiwan
}

\begin{abstract}
Prenyl- and pyrano-xanthones derived from 1,3,6-trihydroxy-9H-xanthen-9-one, a basic backbone of gambogic acid (GA), were synthesized and evaluated for in vitro cytotoxic effects against four human cancer cell lines (KB, KBvin, A549, and DU-145) and anti-inflammatory activity toward superoxide anion generation and elastase release by human neutrophils in response to fMLP/CB. Among them, prenylxanthones 7-13 were generally less active than pyranoxanthones 14-21 in both anticancer and anti-inflammatory assays. Furthermore, two angular 3,3-

dimethypyranoxanthones (16 and 20) showed the greatest and selective activity against the KBvin multidrug resistant (MDR) cell line with $\mathrm{IC}_{50}$ values of 0.9 and $0.8 \mu \mathrm{g} / \mathrm{mL}$, respectively. An angular 3-methyl-3-prenylpyranoxanthone (17) selectively inhibited elastase release with 200 times more potency than phenylmethylsulfonyl fluoride (PMSF), the positive control.
\end{abstract}

\section{Keywords}

1,3,6-Trihydroxy-9H-xanthen-9-one; Gambogic acid (GA); Prenylxanthones; Pyranoxanthones; Cytotoxicity; Anti-inflammatory activity

\footnotetext{
(C) 2012 Elsevier Ltd. All rights reserved.

"Corresponding authors. Tel.: (+886)-4-2205-7153 ext. 1012; fax: (+886)-4-2206-0248 (Y.C.W.); Tel.: (+1)-919-0962-0066; fax: (+1)-919-966-3893 (K.H.L.). yachwu@cmu.edu.tw (Y. C. Wu), khlee@unc.edu (K. H. Lee). .

Publisher's Disclaimer: This is a PDF file of an unedited manuscript that has been accepted for publication. As a service to our customers we are providing this early version of the manuscript. The manuscript will undergo copyediting, typesetting, and review of the resulting proof before it is published in its final citable form. Please note that during the production process errors may be discovered which could affect the content, and all legal disclaimers that apply to the journal pertain.
} 
Gamboge resin, obtained from Garcinia hanburyi in Southeast Asia, has been used as a coloring agent and folk medicine in China. ${ }^{1}$ Gambogic acid (GA) is a natural product isolated from this resin. Its molecular structure features a unique 4oxatricyclo[4.3.1.0]decan-2-one ring system built on a xanthone backbone, and this unique ring system is found only in natural products from the genus Garcinia. $^{2}$ The biogenesis of GA in nature must involve two different pathways, one similar to that of caged benzophenones and the other to simple xanthones. ${ }^{3}$ Ollis and his colleagues reported that GA can be synthesized from normal xanthone. ${ }^{4}$

Aside from its striking chemical architecture, pharmacological studies have revealed that GA possesses potent antitumor activity both in vitro and in vivo, ${ }^{5}$ and GA has entered phase I clinical trials in China for tolerance testing. ${ }^{6}$ In addition, the Garcinia genus is recognized as a rich source of xanthone natural products with high pharmaceutical potential. ${ }^{7} \mathrm{GA}$ contains many functional groups; however, this complex lead compound may have a simpler pharmacophoric moiety buried within its structure. If this pharmacophore can be clearly identified, the resulting simpler molecule may have improved synthetic tractability and be more useful. In order to elucidate the structure-activity relationship (SAR) correlations of GA's basic xanthone skeleton, a retro-synthetic analysis (Figure 1) suggested the design and evaluation of the biological activities of 1,3,6-substituted xanthone derivatives would be reasonable.

Xanthone compounds show potent biological activities, including growth inhibition of various tumor cell lines, ${ }^{8}$ inhibition of human lymphocyte proliferation, ${ }^{9}$ and PKC modulation, ${ }^{10}$ as well as antitumor ${ }^{11}$ and anti-inflammatory activities. ${ }^{12}$ These activities have been associated with the compounds' tricyclic scaffold depending on the nature and/or position of the different substituents. ${ }^{13}$ A previous paper also revealed that several related xanthones, including 1,3,6-trihydroxy-9H-xanthen-9-one, which is the basic skeleton of GA, showed significant activity against sarcoma 180 tumor cells. ${ }^{14}$ Furthermore, recent literature has also shown that prenylated dihydroxyxanthone derivatives exhibited tumor growth inhibitory activity. 3,13

Based on the above results, we designed and synthesized prenylated derivatives structurally related to 1,3,6-trihydroxy-9H-xanthen-9-one, in an effort to find the optimal structural features required for antitumor and anti-inflammatory effects. The synthesized compounds 4-21 have never been isolated as natural products. All new xanthone compounds were assayed for in vitro cytotoxicity against four human cancer cell lines, KB (nasopharyngeal), KBvin (multidrug-resistant nasopharyngeal over-expressing P-gp), A549 (lung), and DU-145 (prostate), and for anti-inflammatory action in terms of superoxide anion generation and elastase release by human neutrophils in response to fMLP/CB.

The synthetic methodologies used to synthesize the xanthone building blocks $\mathbf{4}$ and $\mathbf{5}$, and their derivatives 6-21 are outlined in Schemes 1 and 2. 1,3,6-Trihydroxy-9H-xanthen-9-one was originally prepared by condensation and cyclization reactions between phloroglucinols and appropriately substituted salicylic acids with phosphorus oxychloride-zinc chloride as catalyst. ${ }^{15}$ Later studies provided better results by using a mixture of phosphorus pentoxidemethanesulfonic acid (Eaton's reagent). ${ }^{16}$ Therefore, compounds $\mathbf{4}$ and $\mathbf{5}$ were synthesized in good yields (90-95\%) by the intramolecular oxidative coupling reaction between phloroglucinol (3) and 2,4-dihydroxybenzoic acid (1) or 2-hydroxy-4-methoxybenzoic acid (2), respectively, in the presence of Eaton's reagent, and were used in the next step without purification. Treatment of $\mathbf{4}$ with iodomethane in the presence of $\mathrm{K}_{2} \mathrm{CO}_{3} /$ acetone gave $\mathbf{6}$ $(84 \%)$. Prenylation of 4 with prenyl bromide in the presence of $\mathrm{KOH}$ furnished a mixture of compounds 7-9, which were separated by silica gel column chromatography $(\mathbf{7}: 5.8 \% ; \mathbf{8}$ : $4.2 \% ; 9: 16 \%)$. The $O$-prenylated compounds $10-13$ were prepared by reaction of $\mathbf{4}$ with 
prenyl bromide in the presence of both $\mathrm{KOH}$ and $\mathrm{KI}$, followed again by chromatographic separation (10: $4.2 \%$; 11: 50\%; 12: $5.8 \%$; 13: $10 \%)$.

Cyclization of 4 to the desired linear pyranoxanthones 14 and 15 was accomplished by reactions with prenal (3-methyl-2-butenal) and citral (3,7-dimethyl-2,6-octadienal), respectively, in methanolic calcium hydroxide solution at $\mathrm{rt}$ to afford $85 \%$ and $70 \%$ yields, respectively. For the angular pyranoxanthones 16 and 17, compound 4 was reacted with prenal or citral at $140-150{ }^{\circ} \mathrm{C}$ for $6 \mathrm{~h}$ resulting in yields of $95 \%$ and $93 \%$, respectively. ${ }^{17}$ Dihydrodiolpyranoxanthones 18-21 were prepared by catalytic osmium tetroxide oxidation of 14-17, respectively, using $\mathrm{N}$-methylmorpholine $\mathrm{N}$-oxide to regenerate the oxidizing agent (18: $50 \%$; 19: $45 \%$; 20: $19 \%$; 21: $22 \%) .{ }^{18}$

\section{Cytotoxic activity}

The synthesized compounds 4-21 can be divided into three classes, simple xanthones 4-6, prenylxanthones 7-13, and pyranoxanthones 14-21. Table 1 lists the $\mathrm{IC}_{50}$ values obtained with the test compounds compared to natural GA and the anticancer drug paclitaxel as a positive control. The inhibitory effect of the test drugs on cell viability was measured by the MTT colorimetric method as described previously. ${ }^{19}$

None of the three simple xanthones (4-6) showed significant cytotoxic effects against the KBvin, A549, and DU-145 cancer cell lines. However, the addition of a prenyl group increased the activity. Among the prenylxanthones 7-13, the $C$-prenylxanthones 7-9 IC $_{50}$ $2.8 \sim 6.7 \mu \mathrm{g} / \mathrm{mL})$ were generally more active than the $O$-prenylxanthones 10-13 $\left(\mathrm{IC}_{50} 3.7 \sim\right.$ $>10 \mu \mathrm{g} / \mathrm{mL}$ ) against the three tested cancer cell lines. Compound 10 with one $O$-prenyl moiety was more active than compounds with two $(\mathbf{1 2})$ or three $(\mathbf{1 1}, \mathbf{1 3})$ groups. The DU-145 cell line was most sensitive to these compounds (7-9: IC $_{50} 2.8 \sim 3.9 \mu \mathrm{g} / \mathrm{mL}$ ). Among the pyranoxanthones 14-17, the angular 3-dimethylpyranoxanthone 16 showed a notable $\mathrm{IC}_{50}$ value of $0.9 \mu \mathrm{g} / \mathrm{mL}$ against the KBvin cancer cell line; however, it was much less active against the $\mathrm{KB}, \mathrm{A} 549$, and DU-125 cell lines ( $\mathrm{IC}_{50} 4.8 \sim 5.8 \mu \mathrm{g} / \mathrm{mL}$ ). The corresponding angular 3-methyl-3-prenyl compound (17) and the two linear compounds (14 and 15) exhibited the same range of activity against all four cell lines (IC $504.4 \sim 6.6 \mu \mathrm{g} /$ $\mathrm{mL}$ ). Oxidation of the pyranoxanthones 14-17 to the dihydrodiolpyranoxanthones 18-21 generally resulted in decreased activity, with one exception. Compound 20, the dihydrodiol analog of 16, retained the high activity against the KBvin cancer cell line with an $\mathrm{IC}_{50}$ value of $0.8 \mu \mathrm{g} / \mathrm{mL}$. Overall, the 3 -methyl compounds $(\mathbf{1 4}, \mathbf{1 6}, \mathbf{1 8}$, and $\mathbf{2 0})$ exhibited greater activity than the corresponding 3-prenyl compounds $(\mathbf{1 5}, \mathbf{1 7}, \mathbf{1 9}$, and 21). Although most of the synthetic compounds were inactive or much less active than the natural product GA, compounds $\mathbf{1 6}$ and $\mathbf{2 0}$ were selective for and showed comparable activity with GA against the KBvin cancer cell line, indicating that structural simplification could be a viable option in the design of new chemotherapeutic agents from this compound class. Our research also indicated that pyranoxanthones could have more potent cytotoxic effects than previously discovered, as previous literature showed that prenylated xanthones had weak cytotoxicity $\left(\mathrm{IC}_{50}\right.$ values $\left.50 \sim 80 \mu \mathrm{M}\right){ }^{20}$

\section{Anti-inflammatory activity}

Compounds 4-21 were also evaluated for anti-inflammatory action based on effects against superoxide anion generation and elastase release by human neutrophils in response to fMLP/ $\mathrm{CB}$. The assays were performed using established protocols,${ }^{21}$ which are widely used to identify potential anti-inflammatory compounds. Table 2 lists the results for the test compounds, as well as diphenyleneiodonium (DPI) and phenylmethylsulfonyl fluoride 
(PMSF), included as positive controls for superoxide anion generation and elastase release, respectively.

Xanthone 4 showed a selective inhibitory effect toward superoxide anion generation with an $\mathrm{IC}_{50}$ value of $5.84 \mu \mathrm{g} / \mathrm{mL}$, while compounds 5 and $\mathbf{6}$ exhibited weak activity in both antiinflammatory assays. Among compounds 7-21, prenylxanthones 7-13 demonstrated weaker effects than pyranoxanthones 14-21 in response to superoxide anion generation and elastase release. Linear pyranoxanthone $\mathbf{1 4}$ was the most active compound, with $\mathrm{IC}_{50}$ values of 0.46 and $0.64 \mu \mathrm{g} / \mathrm{mL}$ against superoxide anion generation and elastase release, respectively, and angular pyranoxanthone 17 showed selective anti-inflammatory activity toward elastase release with an $\mathrm{IC}_{50}$ value of $0.49 \mu \mathrm{g} / \mathrm{mL}$. Except for 16, 18, and 20, compounds 14-21 exhibited potent activity toward elastase release and were over 15 -fold more potent than the positive control PMSF.

In this investigation, we prepared a series of 1,3,6-substituted xanthones (4-6), as well as prenyl- and pyrano-xanthone analogs (7-21), ${ }^{22}$ and evaluated SAR for their cytotoxic and anti-inflammatory activities. In conclusion, among all screened compounds, prenylxanthones 7-13 were less active than pyranoxanthones 14-21 in both anticancer and anti-inflammatory assays. Two angular 3,3-dimethylpyranoxanthone analogs (16 and 20) showed notable and selective activity against a multidrug resistant (MDR) cell line (KBvin) with much lower activity against the parent cells (KB). A linear 3,3dimethylpyranoxanthone compound (14) exhibited significant potency in both antiinflammatory assays, and an angular 3-methyl-3-prenylpyranoxanthone compound (17) was 200-fold more potent than PMSF, the positive control, in the elastase release assay.

\section{Acknowledgments}

This investigation was supported by grant CA 17625-32 from the National Cancer Institute, NIH, USA (K. H. Lee), and by grant DOH101-TD-C-111-004 from the Department of Health, Executive Yuan, Taiwan (Y. C. Wu).

\section{References and notes}

1(a). Ollis WD, Redman BT, Sutherland IO, Jewers KJ. Chem. Soc. Chem. Commun. 1969; 15:879.(b) Kumar P, Baslas RK. Herba Hung. 1980; 19:81.

2. Li NG, You QD, Huang XF, Wang JX, Guo QL, Chen XG, Li Y, Li HY. Chin. Chem. Lett. 2007; 18:659.

3. Han Q-B, Yang N-Y, Tian H-L, Qiao C-F, Song J-Z, Chang DC, Chen S-L, Luo KQ, Xu H-X. Phytochemistry. 2008; 69:2187. [PubMed: 18614188]

4. Ollis WD, Ramsay MVJ, Sutherland IO. Tetrahedron. 1965; 21:1453.

5(a). Guo QL, You QD, Wu ZQ, Yuan ST, Zhao L. Acta Pharmacol. Sin. 2004; 25:769. [PubMed: 15169630] (b) Liu W, Guo QL, You QD, Zhao L, Gu HY, Yuan ST. World J. Gastroenterol. 2005; 11:3655. [PubMed: 15968715] (c) Zhao L, Guo QL, You QD, Wu ZQ, Gu HY. Biol. Pharm. Bull. 2004; 27:998. [PubMed: 15256729]

6. Zhou ZT, Wang JW. Chinese J New Drugs. 2007; 16:79.

7. Thoison O, Fahy J, Dumontet V, Chiaroni A, Riche C, Tri MV, Sevenet T. J. Nat. Prod. 2000; 63:441. [PubMed: 10785410]

8. Pedro M, Cerqueira F, Sousa ME, Nascimento MSJ, Pinto M. Bioorg. Med. Chem. 2002; 10:3725. [PubMed: 12413829]

9. Gonzalez MJ, Nascimento MSJ, Cidade HM, Pinto MMM, Kijjoa A, Anantachoke C, Silva AMS, Herz W. Planta Med. 1999; 65:368. [PubMed: 17260263]

10(a). Lu ZX, Hasmeda M, Mahabusarakam W, Ternai B, Ternai PC, Polya GM. Chem. Biol. Interact. 1998; 114:121. [PubMed: 9744560] (b) Jinsart W, Ternai B, Buddhasukh D, Polya GM. Phytochemistry. 1992; 31:3711. [PubMed: 1368866] 
11. Ho CK, Huang YL, Chen CC. Planta Med. 2002; 68:975. [PubMed: 12451486]

12. Nakatani K, Nakahata N, Arakawa T, Yasuda H, Ohizumi Y. Biochem. Pharmacol. 2002; 63:73. [PubMed: 11754876]

13. Castanheiro RAP, Pinto MMM, Silva AMS, Cravo SMM, Gales L, Damas AM, Nazareth N, Nascimento MSJ, Eaton G. Bioorg. Med. Chem. 2007; 15:6080. [PubMed: 17614292]

14. Finnegan RA, Merkel KE, Patel JK. J. Pharm. Sci. 1973; 62:483. [PubMed: 4632437]

15(a). Grover PK, Shah GD, Shah RC. J. Chem. Soc. 1955:3982.(b) Quillinan AJ, Scheinmann F. J. Chem. Soc., Perkin Trans. 1. 1973:1329.

16(a). Zhou T, Shi Q, Chen C-H, Huang L, Ho P, Morris-Natschke SL, Lee K-H. Eur. J. Med. Chem. 2012; 47:86. [PubMed: 22063755] (b) Moreau S, Varache-Lembège M, Larrouture S, Fall D, Neveu A, Deffieux G, Deffieux G, Vercauteren J, Nuhrich A. Eur. J. Med. Chem. 2002; 37:237. [PubMed: 11900868]

17. Mondal M, Puranik VG, Argade NP. J. Org. Chem. 2006; 71:4992. [PubMed: 16776532]

18. Sittisombut C, Boutefnouchet S, Van-Dufat H. Trinh, Tian W, Michel S, Koch M, Tillequin F, Pfeiffer B, Pierre A. Chem. Pharm. Bull. 2006; 54:1113. [PubMed: 16880654]

19. Yen C-T, Nakagawa-Goto K, Hwang T-L, Wu PC, Morris-Natschke SL, Lai W-C, Bastow KF, Chang F-R, Wu Y-C, Lee K-H. Bioorg. Med. Chem. Lett. 2010; 20:1037. [PubMed: 20036537]

20. Castanheiro RAP, Silva AMS, Campos NAN, Nascimento MSJ, Pinto MMM. Pharmaceuticals. 2009; 2:33.

21(a). Hwang T-L, Leu Y-L, Kao S-H, Tang M-C, Chang H-L. Free Radic. Biol. Med. 2006; 41:1433. [PubMed: 17023270] (b) Babior BM, Kipnes RS, Curnutte JT. J. Clin. Invest. 1973; 52:741. [PubMed: 4346473] (c) Sklar LA, McNeil VM, Jesaitis AJ, Painter RG, Cochrane CG. J. Biol. Chem. 1982; 257:5471. [PubMed: 6917847]

22. General: Unless stated otherwise, the chemicals were acquired from commercial sources and used without further purification. All chemicals were purchased from ACROS and Aldrich. Melting points were measured with a Fisher-John melting apparatus without correction. ${ }^{1} \mathrm{H}$ NMR spectra were measured on $300 \mathrm{MHz}$ Varian Gemini 2000 spectrometer. The solvent was $\mathrm{CD}_{3} \mathrm{OD}$ or $\mathrm{CDCl}_{3}$ or DMSO. Mass spectra were measured on PECIEX API 3000 with turbo ion spray source, Agilent-1100 LC/MSD-Trap, or Shimadzu LCMS-IT-TOF with ESI interface. Thin-layer chromatography (TLC) and preparative TLC were performed on precoated silica GF plates purchased from Merck, Inc. Biotage Flash ${ }^{+}$or Isco Companion systems were used for flash chromatography. Silica gel (200-400 mesh) from Aldrich, Inc. was used for column chromatography.1,3,6-Trihydroxy-9H-xanthen-9-one (4). Eaton's reagent $\left(\mathrm{P}_{2} \mathrm{O}_{5}-\mathrm{CH}_{3} \mathrm{SO}_{3} \mathrm{H}\right)(10$ $\mathrm{mL})$ was added slowly to a mixture of 2,4-dihydroxybenzoic acid $(\mathbf{1} ; 155 \mathrm{mg}, 1 \mathrm{mmol})$ and phloroglucinol $(3 ; 126 \mathrm{mg}, 1 \mathrm{mmol})$. The resulting mixture was stirred for $1 \mathrm{~h}$ at $80^{\circ} \mathrm{C}$, cooled to $\mathrm{rt}$, and poured onto ice. After vigorous stirring at ambient temperature for $2 \mathrm{~h}$, thin slurry formed. The solid was collected by filtration, washed with water to adjust the $\mathrm{pH}$ to approximately 6 , and dried under vacuum at $50^{\circ} \mathrm{C}$. The residue was chromatographed on silica gel and eluted successively with hexane-EtOAc (2:3) to give the desired product $(225 \mathrm{mg}, 92 \%)$ as a yellow solid. mp: $158-160{ }^{\circ} \mathrm{C} ;{ }^{1} \mathrm{H}$ NMR $\left(\mathrm{CD}_{3} \mathrm{OD}, 300 \mathrm{MHz}\right): \delta 6.13(1 \mathrm{H}, \mathrm{d}, J=2.1 \mathrm{~Hz}), 6.26(1 \mathrm{H}, \mathrm{d}, J=$ $2.4 \mathrm{~Hz}), 6.72(1 \mathrm{H}, \mathrm{d}, J=2.4 \mathrm{~Hz}), 6.81(1 \mathrm{H}, \mathrm{dd}, J=2.3,8.9 \mathrm{~Hz}), 7.97(1 \mathrm{H}, \mathrm{d}, J=9.0 \mathrm{~Hz}) .1,3-$ Dihydroxy-6-methoxy-9H-xanthen-9-one (5). Under similar conditions to those described for 4, phloroglucinol (3; $126 \mathrm{mg}, 1 \mathrm{mmol})$ and 2,4-dimethoxybenzoic acid $(2 ; 182 \mathrm{mg}, 1 \mathrm{mmol})$ afforded the desired product $(245 \mathrm{mg}, 95 \%)$ as a yellow solid, mp: $135-137{ }^{\circ} \mathrm{C} ;{ }^{1} \mathrm{H}$ NMR $\left(\mathrm{CD}_{3} \mathrm{OD}, 300\right.$ MHz): $\delta 3.89(3 \mathrm{H}, \mathrm{s}), 6.21(1 \mathrm{H}, \mathrm{d}, J=2.1 \mathrm{~Hz}), 6.28(1 \mathrm{H}, \mathrm{d}, J=2.4 \mathrm{~Hz}), 6.70(1 \mathrm{H}, \mathrm{d}, J=2.4 \mathrm{~Hz})$, $6.83(1 \mathrm{H}, \mathrm{dd}, J=2.3,8.9 \mathrm{~Hz}), 7.89(1 \mathrm{H}, \mathrm{d}, J=9.0 \mathrm{~Hz})$.1-Hydroxy-3,6-dimethoxy-9H-xanthen-9one (6). Iodomethane $(100 \mu \mathrm{L}, 1.59 \mathrm{mmol})$ was added to a solution of 4 (130 $\mathrm{mg}, 0.53 \mathrm{mmol})$ and $\mathrm{K}_{2} \mathrm{CO}_{3}(183 \mathrm{mg}, 1.33 \mathrm{mmol})$ in acetone. The resulting solution was stirred at $60^{\circ} \mathrm{C}$ under reflux for $6 \mathrm{~h}$. The cooled solution was filtered and concentrated in vacuo. Purification on a flash column ( $n$-hexane/EtOAc, $85 / 15)$ yielded the desired compound $(231 \mathrm{mg}, 85 \%)$ as a yellow powder. mp: 118-120 ${ }^{\circ} \mathrm{C} ;{ }^{1} \mathrm{H}$ NMR $\left(\mathrm{CDCl}_{3}, 300 \mathrm{MHz}\right): \delta 3.87$ and $3.92(2 \times 3 \mathrm{H}$, each s), 6.31, 6.36, and 6.79 $(3 \times 1 \mathrm{H}$, each s), $6.91(1 \mathrm{H}, \mathrm{d}, J=8.7 \mathrm{~Hz}), 8.11(1 \mathrm{H}, \mathrm{d}, J=8.7 \mathrm{~Hz}), 12.99(1 \mathrm{H}, \mathrm{s})$.Prenylation of 1,3,6-trihydroxy-xanthen-9-one (4). Method 1: Prenyl bromide $(243 \mu \mathrm{L}, 2 \mathrm{mmol})$ was added dropwise over $10 \mathrm{~min}$ to a solution of $4(244 \mathrm{mg}, 1 \mathrm{mmol})$ and $\mathrm{KOH}(112 \mathrm{mg}, 2 \mathrm{mmol})$ in $\mathrm{H}_{2} \mathrm{O}$ at $0{ }^{\circ} \mathrm{C}$ under $\mathrm{N}_{2}$ atmosphere. The resulting mixture was stirred at $0^{\circ} \mathrm{C}$ for $24 \mathrm{~h}$. During this time, a 
yellow solid precipitated from the mixture. The reaction mixture was quenched with $\mathrm{pH} 1(\mathrm{HCl})$ solution and extracted with EtOAc. The combined organic layers were washed with brine, dried over $\mathrm{MgSO}_{4}$, filtered, and concentrated in vacuo. Purification on a flash column yielded 7, 8, and 9 ( $n$-hexane/EtOAc, 95/5) successively.1,3,6-Trihydroxy-2,4-bis(3-methylbut-2-enyl)-9Hxanthen-9-one (7). Yield: 5.8\%; yellow oil; ${ }^{1} \mathrm{H}$ NMR $\left(\mathrm{CD}_{3} \mathrm{OD}, 300 \mathrm{MHz}\right): \delta 1.67$ and $1.89(2 \times$ $6 \mathrm{H}$, each s), $3.34(2 \mathrm{H}, \mathrm{s}), 3.43(2 \mathrm{H}, \mathrm{d}, J=7.2 \mathrm{~Hz}), 5.15-5.19(2 \times 1 \mathrm{H}, \mathrm{m}), 6.69(1 \mathrm{H}, \mathrm{d}, J=2.1 \mathrm{~Hz})$, $6.78(1 \mathrm{H}, \mathrm{dd}, J=2.4,8.7 \mathrm{~Hz}), 7.93(1 \mathrm{H}, \mathrm{d}, J=8.7 \mathrm{~Hz}) .1,3,6$-Trihydroxy-2-(3-methylbut-2enyl)-9H-xanthen-9-one (8). Yield: $4.2 \%$ yield; yellow solid; ${ }^{1} \mathrm{H}$ NMR $\left(\mathrm{CD}_{3} \mathrm{OD}, 300 \mathrm{MHz}\right): \delta$ 1.65 and $1.84(2 \times 3 \mathrm{H}$, each s), $3.35(2 \mathrm{H}, \mathrm{d}, J=6.9 \mathrm{~Hz}), 5.18-5.19(1 \mathrm{H}, \mathrm{m}), 6.14(1 \mathrm{H}, \mathrm{s}), 6.68(1 \mathrm{H}$, d, $J=2.1 \mathrm{~Hz}), 6.76(1 \mathrm{H}, \mathrm{dd}, J=2.1,8.9 \mathrm{~Hz}), 7.89(1 \mathrm{H}, \mathrm{d}, J=9.0 \mathrm{~Hz}) .1,3,6$-Trihydroxy-2,5-bis(3methylbut-2-enyl)-9H-xanthen-9-one (9). Yield: $16 \%$; yellow solid; ${ }^{1} \mathrm{H}$ NMR $\left(\mathrm{CD}_{3} \mathrm{OD}, 300\right.$ $\mathrm{MHz}): \delta 1.67(6 \mathrm{H}, \mathrm{s}), 1.78(2 \times 3 \mathrm{H}, \mathrm{d}, J=4.2 \mathrm{~Hz}), 3.45(2 \mathrm{H}, \mathrm{d}, J=6.3 \mathrm{~Hz}), 3.55(2 \mathrm{H}, \mathrm{d}, J=6.3$ $\mathrm{Hz}), 5.21-5.24(2 \mathrm{H}, \mathrm{m}), 6.19(1 \mathrm{H}, \mathrm{s}), 6.83(1 \mathrm{H}, \mathrm{d}, J=8.7 \mathrm{~Hz}), 7.84(1 \mathrm{H}, \mathrm{d}, J=9.0 \mathrm{~Hz})$. Method 2: To a solution of 4 (244 mg, $1 \mathrm{mmol})$, KI (332 mg, $2 \mathrm{mmol})$, and KOH (112 mg, $2 \mathrm{mmol})$ in DMF at $0{ }^{\circ} \mathrm{C}$ under $\mathrm{N}_{2}$ atmosphere, prenyl bromide $(243 \mu \mathrm{L}, 2 \mathrm{mmol})$ was added dropwise over 10 min. The resulting mixture was then treated under conditions similar to Method 1. Purification on a flash column yielded a mixture of $\mathbf{1 0}+\mathbf{1 1}+\mathbf{1 2}$ ( $n$-hexane/EtOAc, 95/5) and pure $\mathbf{1 3}$ ( $n$-hexane/ EtOAc, 90/10). The components of the mixture were then separated by preparative TLC ( $n$ hexane/EtOAc, 4/1).1,6-Dihydroxy-3-(3-methylbut-2-enyloxy)-9H-xanthen-9-one (10). Yield: 4.2\%; yellow oil; ${ }^{1} \mathrm{H}$ NMR $\left(\mathrm{CDCl}_{3}, 300 \mathrm{MHz}\right): \delta 1.67(2 \times 3 \mathrm{H}$, each s), $3.30(2 \mathrm{H}, \mathrm{s}), 3.43(2 \mathrm{H}, \mathrm{d}$, $J=7.2 \mathrm{~Hz}), 5.15-5.19(1 \mathrm{H}, \mathrm{m}), 6.13(1 \mathrm{H}, \mathrm{d}, J=2.1 \mathrm{~Hz}), 6.26(1 \mathrm{H}, \mathrm{d}, J=2.4 \mathrm{~Hz}), 6.69(1 \mathrm{H}, \mathrm{d}, J=$ $2.1 \mathrm{~Hz}), 6.78(1 \mathrm{H}, \mathrm{dd}, J=2.4,8.7 \mathrm{~Hz}), 7.93(1 \mathrm{H}, \mathrm{d}, J=8.7 \mathrm{~Hz}) .1$-Hydroxy-2-(3-methylbut-2enyl)-3,6-bis(3-methylbut-2-enyloxy)-9H-xanthen-9-on e (11). Yield: 50\%; yellow solid; ${ }^{1} \mathrm{H}$ NMR $\left(\mathrm{CDCl}_{3}, 300 \mathrm{MHz}\right): \delta 1.77-1.82(12 \mathrm{H}, \mathrm{m}), 1.85-1.90(6 \mathrm{H}, \mathrm{m}), 4.55-4.61(6 \mathrm{H}, \mathrm{m}), 5.49-5.51(4 \mathrm{H}$, $\mathrm{m}), 6.35(1 \mathrm{H}, \mathrm{d}, J=1.8 \mathrm{~Hz}), 6.40(1 \mathrm{H}, \mathrm{d}, J=1.8 \mathrm{~Hz}), 6.78(1 \mathrm{H}, \mathrm{d}, J=1.8 \mathrm{~Hz}), 6.89(1 \mathrm{H}, \mathrm{dd}, J=$ $2.1,9.0 \mathrm{~Hz}), 8.08(1 \mathrm{H}, \mathrm{d}, J=8.7 \mathrm{~Hz}), 13.19(1 \mathrm{H}, \mathrm{s}) .1$-Hydroxy-3,6-bis(3-methylbut-2enyloxy)-9H-xanthen-9-one (12). Yield: 5.8\%; yellow solid; ${ }^{1} \mathrm{H}$ NMR $\left(\mathrm{CDCl}_{3}, 300 \mathrm{MHz}\right): \delta$ 1.77-1.82 (12H, m), 4.55-4.61 (4H, m), 5.49-5.51 (2H, m), $6.30(1 \mathrm{H}, \mathrm{d}, J=1.8 \mathrm{~Hz}), 6.35(1 \mathrm{H}, \mathrm{d}$, $J=1.8 \mathrm{~Hz}), 6.78(1 \mathrm{H}, \mathrm{d}, J=1.8 \mathrm{~Hz}), 6.89(1 \mathrm{H}, \mathrm{dd}, J=2.1,9.0 \mathrm{~Hz}), 8.08(1 \mathrm{H}, \mathrm{d}, J=8.7 \mathrm{~Hz}), 13.27$ (1H, s).1,3,6-Tris(3-methylbut-2-enyloxy)-9H-xanthen-9-one (13). Yield: $10 \%$; yellow solid; ${ }^{1} \mathrm{H}$ NMR (CD $\left.{ }_{3} \mathrm{OD}, 300 \mathrm{MHz}\right): \delta 1.78(18 \mathrm{H}, \mathrm{s}), 4.57(4 \mathrm{H}, \mathrm{t}, J=6.5 \mathrm{~Hz}), 4.64(2 \mathrm{H}, \mathrm{d}, J=6.3 \mathrm{~Hz})$, $5.44-5.52(3 \mathrm{H}, \mathrm{m}), 6.28(1 \mathrm{H}, \mathrm{d}, J=1.8 \mathrm{~Hz}), 6.38(1 \mathrm{H}, \mathrm{d}, J=2.1 \mathrm{~Hz}), 6.73(1 \mathrm{H}, \mathrm{d}, J=2.1 \mathrm{~Hz})$, $6.82(1 \mathrm{H}, \mathrm{dd}, J=2.1,8.7 \mathrm{~Hz}), 7.97(1 \mathrm{H}, \mathrm{d}, J=8.7 \mathrm{~Hz}) .5$,9-Dihydroxy-2,2-dimethylpyrano[3,2$b$ ]xanthen-6(2H)-one (14). Prenal $(480 \mu \mathrm{L}, 5 \mathrm{mmol})$ was added to a stirring mixture of 4 (244 $\mathrm{mg}$, $1 \mathrm{mmol})$ and $\mathrm{Ca}(\mathrm{OH})_{2}(150 \mathrm{mg}, 2 \mathrm{mmol})$ in $\mathrm{MeOH}$ at rt. After continued stirring for $36 \mathrm{~h}$ at $\mathrm{rt}$, $\mathrm{MeOH}$ was removed under vacuum, and the reaction mixture was diluted with EtOAc. The organic layer was washed with $2 \mathrm{~N} \mathrm{HCl}$, water, and brine, and dried over $\mathrm{MgSO}_{4}$. Purification on a flash column ( $n$-hexane/EtOAc, 90/10) afforded the desired compound $(\mathbf{1 4}, 85 \%)$ as a yellow solid. mp: $228-230{ }^{\circ} \mathrm{C} ;{ }^{1} \mathrm{H} \mathrm{NMR}\left(\mathrm{CDCl}_{3}+\mathrm{CD}_{3} \mathrm{OD}, 300 \mathrm{MHz}\right): \delta 1.43(6 \mathrm{H}, \mathrm{s}), 5.63$ and $6.27(2 \times$ $1 \mathrm{H}$, each s), $6.65(1 \mathrm{H}, \mathrm{d}, J=10.2 \mathrm{~Hz}), 6.74(1 \mathrm{H}, \mathrm{d}, J=1.8 \mathrm{~Hz}), 6.81(1 \mathrm{H}, \mathrm{dd}, J=2.3,8.9 \mathrm{~Hz}), 7.98$ $(1 \mathrm{H}, \mathrm{d}, J=8.7 \mathrm{~Hz}) .5,9-$ Dihydroxy-2-methyl-2-(4-methylpent-3-enyl)pyrano[3,2- $b]$ xanthen-6(2H)one (15). Under conditions similar to those described for the preparation of 14, compound 4 (244 $\mathrm{mg}, 1 \mathrm{mmol})$ and citral $(860 \mu \mathrm{L}, 5 \mathrm{mmol})$ afforded $15(70 \%)$ as a yellow solid. ${ }^{1} \mathrm{H} \mathrm{NMR}\left(\mathrm{CDCl}_{3}\right.$, $300 \mathrm{MHz}): \delta 1.35,1.56$, and $1.64(3 \times 3 \mathrm{H}$, each s), 1.76-1.83 $(2 \mathrm{H}, \mathrm{m}), 2.06-2.10(2 \mathrm{H}, \mathrm{m}), 5.07-$ $5.10(1 \mathrm{H}, \mathrm{m}), 5.52(1 \mathrm{H}, \mathrm{d}, J=10.2 \mathrm{~Hz}), 6.27(1 \mathrm{H}, \mathrm{s}), 6.72-6.85(3 \mathrm{H}, \mathrm{m}), 8.05(1 \mathrm{H}, \mathrm{d}, J=9.0 \mathrm{~Hz})$, $13.21(1 \mathrm{H}, \mathrm{s}) .6,10$-Dihydroxy-3,3-dimethylpyrano[2,3-c]xanthen-7(3H)-one (16). A stirring mixture of $4(244 \mathrm{mg}, 1 \mathrm{mmol})$ and prenal $(960 \mu \mathrm{L}, 10 \mathrm{mmol})$ was heated at $140-150^{\circ} \mathrm{C}$ for $6 \mathrm{~h}$. After cooling, the residue was purified on a flash column ( $n$-hexane/EtOAc, 90/10) to afford $\mathbf{1 6}$ (295 mg, 95\%) as a yellow powder. ${ }^{1} \mathrm{H}$ NMR $\left(\mathrm{CDCl}_{3}+\mathrm{CD}_{3} \mathrm{OD}, 300 \mathrm{MHz}\right): \delta 1.48(6 \mathrm{H}, \mathrm{s}), 5.66$ $(1 \mathrm{H}, \mathrm{d}, J=9.6 \mathrm{~Hz}), 6.16(1 \mathrm{H}, \mathrm{s}), 6.79-6.87(3 \mathrm{H}, \mathrm{m}), 8.02(1 \mathrm{H}, \mathrm{d}, J=8.7 \mathrm{~Hz}) .6,10-$ Dihydroxy-3methyl-3-(4-methylpent-3-enyl)pyrano[2,3-c]xanthen-7(3H)-one (17). Under conditions similar to those described for the preparation of 16, compound $4(244 \mathrm{mg}, 1 \mathrm{mmol})$ and citral $(1.7 \mathrm{~mL}, 10$ mmol) afforded $17(351.9 \mathrm{mg}, 93 \%)$ as an amber-brown oil. ${ }^{1} \mathrm{H} \mathrm{NMR}\left(\mathrm{CDCl}_{3}, 300 \mathrm{MHz}\right): \delta 1.43$, 1.56 and $1.64(3 \times 3 \mathrm{H}$, each s), 1.78-1.83 $(2 \mathrm{H}, \mathrm{m}), 2.05-2.13(2 \mathrm{H}, \mathrm{m}), 5.05-5.10(1 \mathrm{H}, \mathrm{m}), 5.53$ $(1 \mathrm{H}, \mathrm{d}, J=10.2 \mathrm{~Hz}), 6.23(1 \mathrm{H}, \mathrm{s}), 6.80-6.86(3 \mathrm{H}, \mathrm{m}), 8.09(1 \mathrm{H}, \mathrm{d}, J=9.0 \mathrm{~Hz}), 13.07(1 \mathrm{H}, \mathrm{s})$. 3,4,5,9-Tetrahydroxy-2,2-dimethyl-3,4-dihydropyrano[3,2- $b]$ xanthen-6(2H)-one (18). Compound 14 (84 mg, $0.22 \mathrm{mmol}$ ) was added to a solution of osmium tetroxide (2.5\% in 2-methyl-2- 
propanol, $210 \mu \mathrm{L}$ ) and 4-methylmorpholine $N$-oxide monohydrate (30 mg, $0.22 \mathrm{mmol})$ in $t$ $\mathrm{BuOH}-\mathrm{THF}-\mathrm{H}_{2} \mathrm{O}(10: 3: 1,14 \mathrm{~mL})$. The reaction mixture was stirred at $\mathrm{rt}$ for $48 \mathrm{~h}$. After addition of saturated aqueous sodium bisulfate solution $(30 \mathrm{~mL})$, the mixture was stirred for $1 \mathrm{~h}$ and then extracted with $\mathrm{CH}_{2} \mathrm{Cl}_{2}$. The combined organic layers were dried with $\mathrm{MgSO}_{4}$, filtered, and evaporated in vacuo. Purification on a flash column $\left(\mathrm{CH}_{2} \mathrm{Cl}_{2} / \mathrm{MeOH}, 98 / 2\right)$ gave the desired product (172.2 mg, 50\%) as a yellow oil. ${ }^{1} \mathrm{H}$ NMR (DMSO, $\left.300 \mathrm{MHz}\right): \delta 1.41(6 \mathrm{H}, \mathrm{s}), 3.67(1 \mathrm{H}$, $\mathrm{d}, J=8.5 \mathrm{~Hz}), 5.02(1 \mathrm{H}, \mathrm{d}, J=8.3 \mathrm{~Hz}), 6.68(1 \mathrm{H}, \mathrm{d}, J=10.2 \mathrm{~Hz}), 6.77(1 \mathrm{H}, \mathrm{d}, J=1.8 \mathrm{~Hz}), 6.80$ $(1 \mathrm{H}, \mathrm{dd}, J=2.3,8.9 \mathrm{~Hz}), 7.94(1 \mathrm{H}, \mathrm{d}, J=8.7 \mathrm{~Hz}) .3,4,5,9-T e t r a h y d r o x y-2-m e t h y l-2-(4-$ methylpent-3-enyl)-3,4-dihydropyrano[3,2-b]xa nthen-6(2H)-one (19). Using similar conditions to those described for the preparation of $\mathbf{1 8}$, compound 15 (150 mg, $0.4 \mathrm{mmol}$ ) afforded 19 (186 mg, $45 \%$ ) as a yellow solid. ${ }^{1} \mathrm{H}$ NMR (DMSO, $\left.300 \mathrm{MHz}\right): \delta 1.35,1.56$ and $1.64(3 \times 3 \mathrm{H}$, each s), 1.76-1.83 (2H, m), 2.06-2.10 (2H, m), 5.07-5.10 (1H, m), $3.37(1 \mathrm{H}, \mathrm{d}, J=8.5 \mathrm{~Hz}), 5.05(1 \mathrm{H}, \mathrm{d}, J$ $=8.7 \mathrm{~Hz}), 5.52(1 \mathrm{H}, \mathrm{d}, J=10.2 \mathrm{~Hz}), 6.27(1 \mathrm{H}, \mathrm{s}), 6.72-6.85(3 \mathrm{H}, \mathrm{m}), 8.05(1 \mathrm{H}, \mathrm{d}, J=9.0 \mathrm{~Hz})$, $13.17(1 \mathrm{H}, \mathrm{s}) .1,2,6,10$-Tetrahydroxy-3,3-dimethyl-2,3-dihydropyrano[2,3-c]xanthen-7(1H)-one (20). Under similar conditions to those described for the preparation of $\mathbf{1 8}$, compound $\mathbf{1 6}$ (58.6 $\mathrm{mg}, 0.19 \mathrm{mmol}$ ) afforded $20(172 \mathrm{mg}, 50 \%)$ as an off-white solid. ${ }^{1} \mathrm{H}$ NMR (DMSO, $\left.300 \mathrm{MHz}\right): \delta$ $1.44(6 \mathrm{H}, \mathrm{s}), 3.26(1 \mathrm{H}, \mathrm{d}, J=8.7 \mathrm{~Hz}), 5.03(1 \mathrm{H}, \mathrm{d}, J=8.4 \mathrm{~Hz}), 6.14(1 \mathrm{H}, \mathrm{s}), 6.79-6.87(3 \mathrm{H}, \mathrm{m})$, $8.03(1 \mathrm{H}, \mathrm{d}, J=8.7 \mathrm{~Hz}) .1,2,6,10$-Tetrahydroxy-3-methyl-3-(4-methylpent-3-enyl)-2,3dihydropyrano[2,3-c]xa nthen-7(1H)-one (21). Under similar conditions to those described for the preparation of 18, compound $17(84 \mathrm{mg}, 0.22 \mathrm{mmol})$ afforded $21(194 \mathrm{mg}, 47 \%)$ as yellow oil. ${ }^{1} \mathrm{H}$ NMR (DMSO, $300 \mathrm{MHz}): \delta 1.45,1.60$ and $1.64(3 \times 3 \mathrm{H}$, each s), 1.78-1.83 $(2 \mathrm{H}, \mathrm{m}), 2.05-2.13$ $(2 \mathrm{H}, \mathrm{m}), 3.78(1 \mathrm{H}, \mathrm{d}, J=8.7 \mathrm{~Hz}), 5.04(1 \mathrm{H}, \mathrm{d}, J=8.7 \mathrm{~Hz}), 5.53(1 \mathrm{H}, \mathrm{d}, J=10.2 \mathrm{~Hz}), 6.23(1 \mathrm{H}, \mathrm{s})$, 6.80-6.86 (3H, m), $8.09(1 \mathrm{H}, \mathrm{d}, J=9.0 \mathrm{~Hz}), 13.10(1 \mathrm{H}, \mathrm{s})$. 

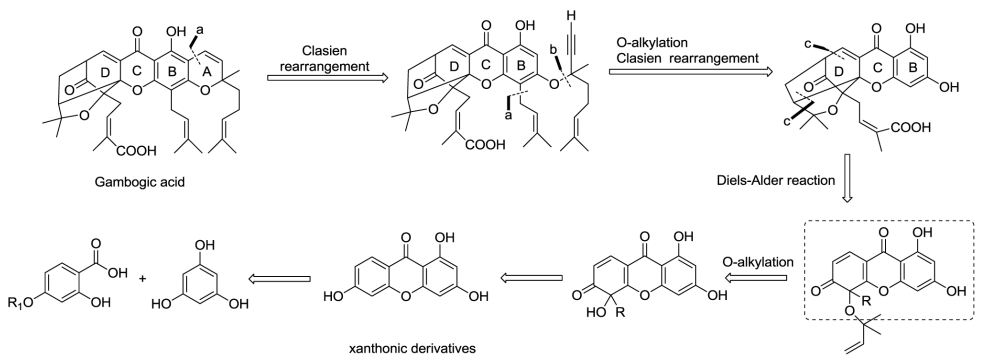

Figure 1.

The retrosynthesis of gambogic acid. 

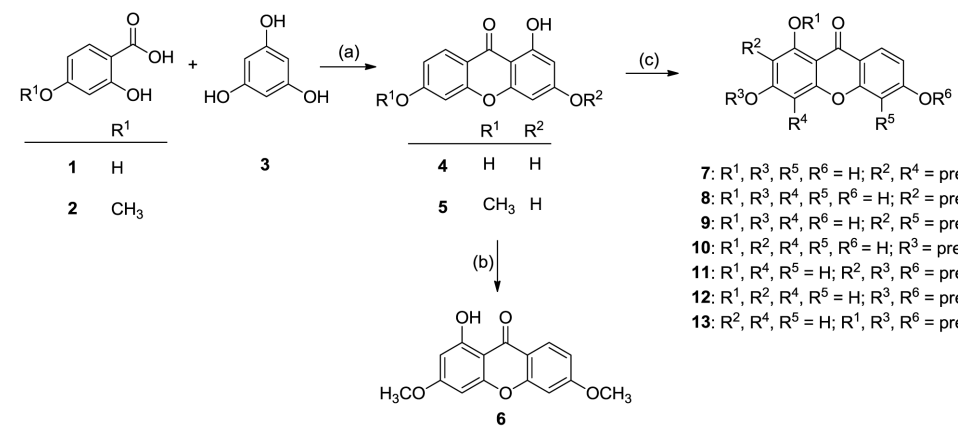

7: $R^{1}, R^{3}, R^{5}, R^{6}=H ; R^{2}, R^{4}=$ prenyl 8: $R^{1}, R^{3}, R^{4}, R^{5}, R^{6}=H ; R^{2}=$ prenyl 9: $R^{1}, R^{3}, R^{4}, R^{6}=H ; R^{2}, R^{5}=$ prenyl 10: $R^{1}, R^{2}, R^{4}, R^{5}, R^{6}=H ; R^{3}=$ prenyl 11: $R^{1}, R^{4}, R^{5}=H ; R^{2}, R^{3}, R^{6}=$ prenyl 12: $R^{1}, R^{2}, R^{4}, R^{5}=H ; R^{3}, R^{6}=$ prenyl

Scheme 1.

The synthesis of compounds 4-13.

Reagents: (a) $\mathrm{P}_{2} \mathrm{O}_{5}-\mathrm{CH}_{3} \mathrm{SO}_{3} \mathrm{H}, 80{ }^{\circ} \mathrm{C}, 1 \mathrm{~h}\left(4: 92 \%\right.$; 5: 95\%). (b) $\mathrm{MeI}, \mathrm{K}_{2} \mathrm{CO}_{3}$, acetone, reflux, $60{ }^{\circ} \mathrm{C}$, overnight $(\mathbf{6}, 85 \%)$. (c) (i) $\mathrm{KOH}$, prenyl bromide, $\mathrm{H}_{2} \mathrm{O}, 0^{\circ} \mathrm{C}, 24 \mathrm{~h}(7: 5.8 \%$; 8: $4.2 \%$; 9: $16 \%$;). (ii) $\mathrm{KOH}, \mathrm{KI}$, prenyl bromide, DMF, $0{ }^{\circ} \mathrm{C}, 24 \mathrm{~h}$ (10: $4.2 \%$; 11: 50\%; 12: $5.8 \% ; 13: 10 \%)$. 


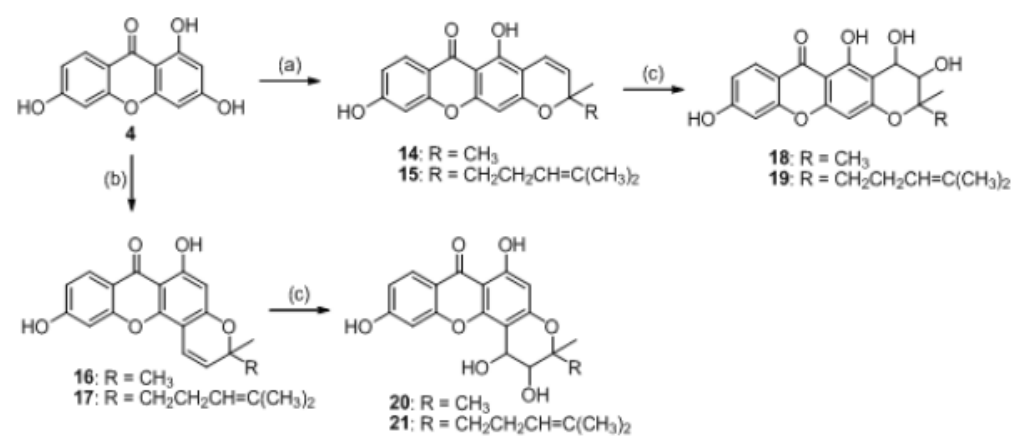

Scheme 2.

The synthesis of pyranoxanthones 14-21.

Reagents and conditions: (a) prenal/citral, $\mathrm{Ca}(\mathrm{OH})_{2}, \mathrm{MeOH}, \mathrm{rt}, 36 \mathrm{~h}(\mathbf{1 4}: 85 \% ; 15: 70 \%)$. (b) $\mathrm{prenal} /$ citral, $140-150^{\circ} \mathrm{C}, 6 \mathrm{~h}\left(\mathbf{1 6}: 95 \%\right.$; 17: 93\%). (c) $\mathrm{OsO}_{4}, \mathrm{NMMO}, t$-BuOH-THF-H $\mathrm{O}_{2} \mathrm{O}$ (10:3:1), rt, 48 h (18: 85\%; 19: 70\%; 20: 19\%; 21: 22\%). 
Table 1

Cytotoxicity of compounds $\mathbf{4 - 2 1}$.

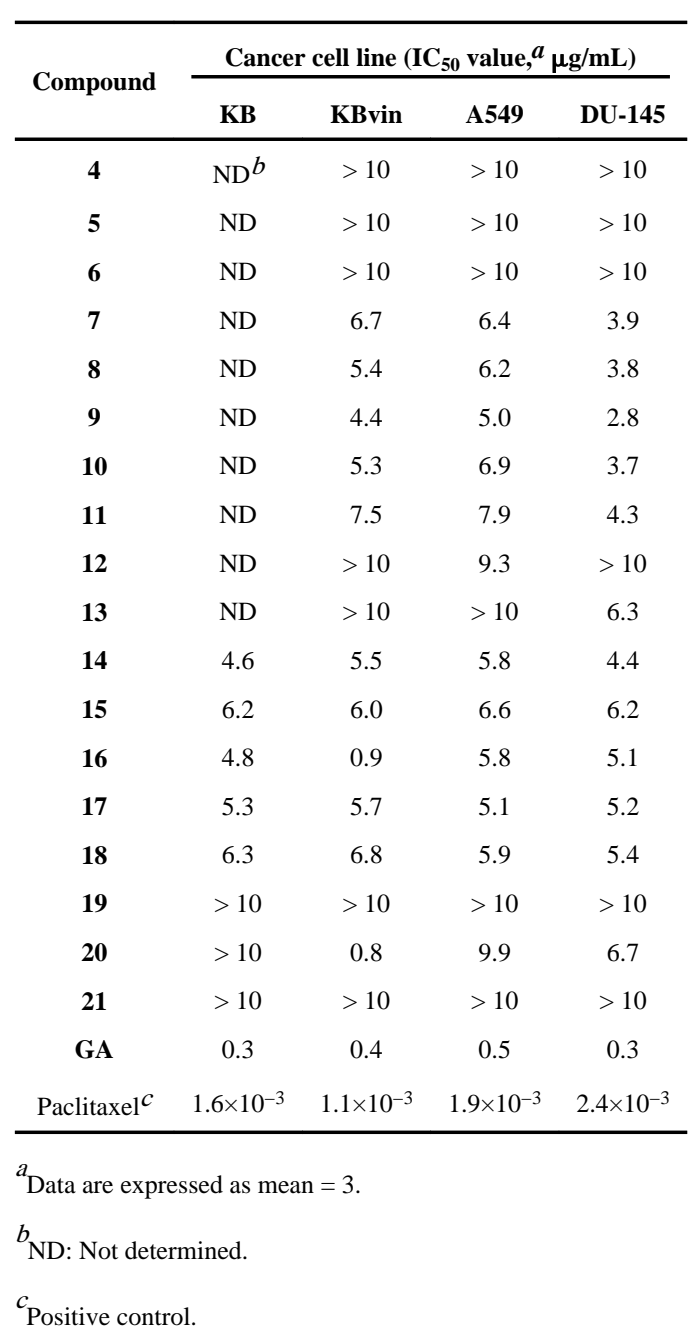


Table 2

Inhibitory effects of compounds on superoxide anion generation and elastase release by human neutrophils in response to fMLP/CB.

\begin{tabular}{|c|c|c|}
\hline \multirow{2}{*}{ Compound } & Superoxide anion & Elastase release \\
\hline & $\mathrm{IC}_{50}(\mu \mathrm{g} / \mathrm{mL})^{a}$ or $(\operatorname{Inh} \%)^{a}$ & $\operatorname{IC}_{50}(\mu \mathrm{g} / \mathrm{mL})^{a}$ or $(\operatorname{Inh} \%)^{a}$ \\
\hline 4 & $5.84 \pm 0.75$ & $(16.0 \pm 6.57)$ \\
\hline 5 & $(45.2 \pm 3.22)$ & $(23.6 \pm 1.24)$ \\
\hline 6 & $(31.3 \pm 3.88)^{* * *}$ & $(26.5 \pm 5.04)^{* * *}$ \\
\hline 7 & $(101 \pm 5.47)^{b}$ & $(102 \pm 1.94)^{b}$ \\
\hline 8 & $(45.2 \pm 0.43)$ & $(56.3 \pm 3.42)$ \\
\hline 9 & $(31.6 \pm 0.21)$ & $(67.2 \pm 5.65)$ \\
\hline 10 & $20.2 \pm 0.52$ & $(4.21 \pm 5.22)$ \\
\hline 11 & $(10.1 \pm 2.73)$ & $(7.56 \pm 3.98)$ \\
\hline 12 & $(5.32 \pm 0.12)$ & $(13.3 \pm 5.32)$ \\
\hline 13 & $(64.3 \pm 0.22)$ & $(54.9 \pm 5.73)$ \\
\hline 14 & $0.46 \pm 0.05$ & $0.64 \pm 0.22$ \\
\hline 15 & $5.43 \pm 0.82$ & $8.75 \pm 0.69$ \\
\hline 16 & $3.97 \pm 0.45$ & $(18.4 \pm 1.44)$ \\
\hline 17 & $35.6 \pm 6.83^{c^{* * *}}$ & $0.49 \pm 0.10$ \\
\hline 18 & $2.49 \pm 0.38$ & $(35.3 \pm 1.59)^{* * *}$ \\
\hline 19 & $4.30 \pm 0.33$ & $5.75 \pm 0.17$ \\
\hline 20 & $(8.63 \pm 6.95)$ & $(6.37 \pm 5.04)$ \\
\hline 21 & $2.56 \pm 0.28$ & $8.46 \pm 0.73$ \\
\hline $\mathrm{DPI}^{d}$ & $0.7 \pm 0.4$ & \\
\hline $\mathrm{PMSF}^{d}$ & & $131 \pm 2.91$ \\
\hline
\end{tabular}

${ }^{a}$ IC 50 represents the $50 \%$ inhibitory concentration of the compound. If $50 \%$ inhibition was not reached at any test dose, the percentage of inhibition obtained at a test dose of $10 \mu \mathrm{g} / \mathrm{mL}$ is given in parentheses $(\mathrm{Inh} \%)$. Results are presented as mean \pm S.E.M. $(\mathrm{n}=3-5)$.

***

$p<0.001$ compared with the control value.

$b_{7}$ alone elicited superoxide anion generation and elastase release by human neutrophils in the absence of fMLP/CB.

$c_{17}$ induced superoxide generation in the pretreatment of cytochalasin B.

${ }^{d}$ DPI and PMSF were used as positive controls. 